\title{
Central Blood Pressure in Young Kendo Athletes: Implications of Combined Anaerobic and Strength Training
}

\author{
Masaki Yoshioka ${ }^{1}$, Kaname Tagawa $a^{1,2}$, Yuriko Tochigi ${ }^{1}$, Tomohito Sato ${ }^{1}$, Jiyeon Park ${ }^{1, \dagger}$, Reiko Momma ${ }^{1}$, \\ Youngju Choi ${ }^{3,5, \mathbb{D}}$, Jun Sugawara ${ }^{4,5,(\mathbb{D}), \text { Seiji Maeda }{ }^{5, *} \text { (D) }}$ \\ ${ }^{1}$ Graduate School of Comprehensive Human Sciences, University of Tsukuba, 1-1-1 Tennodai, Tsukuba, Ibaraki 305-8574, Japan \\ ${ }^{2}$ Graduate School of Education, Miyagi University of Education, 149, Aramaki-aza-Aoba, Aoba, Sendai, Miyagi 980-0845, Japan \\ ${ }^{3}$ Institute of Sport \& Art Convergence, Inha University, 100 Inha-ro, Michuhol-gu, Incheon 22212, South Korea \\ ${ }^{4} \mathrm{Human}$ Informatics and Interaction Research Institute, National Institute of Advanced Industrial Science and Technology, 1-1-1 Higashi, \\ Tsukuba, Ibaraki, Japan \\ ${ }^{5}$ Faculty of Health and Sport Sciences, University of Tsukuba, 1-1-1 Tennodai, Tsukuba, Ibaraki 305-8574, Japan
}

\section{ARTICLE INFO}

\section{Article History}

Received 19 August 2020

Accepted 09 December 2020

\section{Keywords}

Central blood pressure

martial art

stroke volume

anaerobic training

resistance training

\begin{abstract}
Background: Exercise training-induced adaptation of central Blood Pressure (BP) depends on exercise mode. Kendo, a traditional Japanese martial art, is a unique exercise mode because its training encompasses anaerobic and resistance training components. However, the effects of habitual kendo training on central BP have not been established.
\end{abstract}

Objective: The aim of this study was to compare the central BP of high-level kendo athletes and age-matched controls without exercise habits.

Methods: Thirty-six young university kendo athletes (the kendo athlete group) and 29 young sedentary individuals (the control group) participated in this cross-sectional study. Central hemodynamics were estimated from carotid arterial waveforms via a generalized transfer function. Stroke volume was computed from brachial arterial waveforms using the Modelflow method.

Results: Central systolic BP and Central Pulse Pressure (cPP) were higher in the kendo athlete group than in the control group (both, $p<0.01$ ). Central diastolic BP did not differ between the two groups. Stroke Volume Index (SVI; stroke volume adjusted for body surface area) and the maximum rate of aortic pressure rise during systole ( $\left.\mathrm{d} P / \mathrm{d} T_{\max }\right)$, which reflects left ventricle contractility, were significantly higher in the kendo athlete group than in the control group $(p<0.05)$, and these parameters showed a significant positive correlation with cPP (SVI: $r=0.34, p<0.01 ; \mathrm{dP} / \mathrm{d} T_{\max }: r=0.79, p<0.01$ ).

Conclusion: These results suggest that habitual kendo training may increase central pulse pressure through increases in left ventricular systolic function.

\section{HIGHLIGHTS}

- Kendo is ideal for investigating of training adaptation since it induces the characteristics of anaerobic exercise training and resistance training; that is, kendo athletes had both high anaerobic capacity and high muscle strength compared with sedentary controls.

- In the present study, central blood pressure (central systolic blood pressure and central pulse pressure) was significantly higher in the kendo athlete group than in the control group.

- Stroke Volume Index (SVI; stroke volume adjusted for body surface area) and the maximum rate of aortic pressure rise during systole $\left(\mathrm{d} P / \mathrm{d} T_{\max }\right)$ but not augmented pressure in the kendo athlete group were also significantly higher than those in the control group.

- In addition, SVI and $\mathrm{d} P / \mathrm{d} T_{\max }$ showed a significant positive correlation with central pulse pressure.

- These results suggest that combined anaerobic exercise and muscular strength training may increase central blood pressure through increases in left ventricular systolic function.

(C) 2021 The Authors. Publishing services by Atlantis Press International B.V. This is an open access article distributed under the CC BY-NC 4.0 license (http://creativecommons.org/licenses/by-nc/4.0/).

\section{INTRODUCTION}

Central arteries (e.g., carotid artery and aorta), which have high elasticity and extensibility, are passively extended during systole,

"Corresponding author.Email: maeda.seiji.gn@u.tsukuba.ac.jp

Faculty of Health and Sport Sciences, University of Tsukuba, 1-1-1 Tennodai, Tsukuba, Ibaraki 305-8574, Japan

Peer review under responsibility of the Association for Research into Arterial Structure and Physiology

Data availability statement: The authors confirm that the data supporting the findings of this study are available within the article. and approximately $50 \%$ of the stroke volume from the left ventricle is stored inside the artery. In diastole, the stretched arterial wall returns to its original shape and sends the stored blood to the periphery $[1,2]$. This is called "Windkessel function" and it plays an important role in maintaining the central circulation [3]. A previous study reported that central Blood Pressure (BP) was closely related to the Windkessel function [4].

Habitual exercise (including aerobic exercise and muscular strength training) has been recommended for the improvement of body composition, muscle function, glucose metabolism, and health-related 
quality of life [5]. It is well established that aerobic exercise training decreases Central Systolic BP (cSBP) [6]. Interestingly, anaerobic exercise training (i.e., high-intensity interval training) has a more effective central BP-lowering effect than moderate continuous exercise training, a general aerobic exercise training $[7,8]$. In contrast to the beneficial effects of anaerobic exercise, cSBP and Central Pulse Pressure (cPP) in resistance-trained athletes are higher than in sedentary controls [9]. These reports suggest that exercise training-induced adaptation of central BP may differ depending on the exercise training type. However, how combined anaerobic exercise and muscular strength training changes central BP is not fully understood.

Kendo is an individual combative martial art in which two opponents compete to score valid points on each other. The kendo population in Japan has reached about 2 million, and 56 countries participated in the World Kendo Championships in 2018 [10,11]. The number of kendo athletes worldwide is expected to increase. Kendo athletes require high muscle strength to swing the shinai (bamboo sword) [12]. In addition, kendo athletes carry out kakari$k e i k o$, a practice method in which the trainee practices striking the motodachi (a person acting as an instructor) with all their might until the trainee becomes physically and mentally exhausted [13]. Katsuki et al. [14] reported that university kendo athletes had both high anaerobic capacity and high muscle strength compared with the sedentary controls. This study suggested that the adaptation of central BP in young kendo athletes may be affected by combined anaerobic exercise and muscular strength training. However, the effects of regular kendo training on central BP have not been established. Because more time may be required for the development of central hemodynamics, a cross-sectional study analyzing central BP in young kendo athletes may shed light on this question.

The purpose of this study was to compare the central BP of highlevel kendo athletes and age-matched controls without exercise habits. In this cross-sectional study, we measured central hemodynamics including central BP in university kendo athletes and agematched healthy adults without exercise habits.

\section{MATERIALS AND METHODS}

\subsection{Subjects}

A total of 36 young kendo athletes (23 men and 13 women, aged 18-23 years; the kendo athlete group) and 29 young, healthy, sedentary, age-matched adults (17 men and 12 women, aged 18-25 years; the control group) participated in this study. All kendo athletes were high-level university kendo athletes belonging to the university kendo club, and the durations of their kendo careers were $13 \pm 2$ years (range, 6-17 years). The training intensity and practice frequency were at least $120 \mathrm{~min} /$ day and six sessions/week, respectively. The control group included participants who had not been involved in any regular exercise program for at least 1 year. All participants were free from hypertensive, cardiovascular, and metabolic diseases as assessed by medical history, and none of the participants was taking cardiovascular or blood pressure medications. The participants were fully informed of the purposes, risks, and discomforts associated with the experiment before signing written informed consent. All procedures were approved by the Ethics Committee of the University of Tsukuba (approval no. Tai 28-134).

\subsection{Procedures}

This was a cross-sectional study. Before each test, participants abstained from caffeine and alcohol and fasted for at least $12 \mathrm{~h}$ (only water was allowed). All participants were studied at least $12 \mathrm{~h}$ after the last exercise to avoid the acute effect of exercise. Measurements were obtained in a quiet temperature-controlled room $\left(24-26^{\circ} \mathrm{C}\right)$. Hemodynamic parameters were measured after sitting at rest for at least $20 \mathrm{~min}$.

\subsection{Measurements}

\subsubsection{Aortic pulse wave estimations}

Using validated generalized transfer function-based data analysis software (SphygmoCor, AtCor Medical, Sydney, Australia), cSBP, $\mathrm{cPP}$, and maximal rate of pressure increase $\left(\mathrm{d} P / \mathrm{d} T_{\max }\right.$, an index of left ventricle systolic function) were estimated from carotid arterial waveforms, and the estimated waveforms were calibrated and scaled using each participant's resting Brachial Diastolic BP (bDBP) and Mean BP (MBP). The heart rate-corrected (at 75 beats/min) Augmentation Index (AIx) was calculated by dividing the Augmented Pressure (AP) (the difference between the early and late systolic peaks of the synthesized aortic pressure waveform) by cPP. The daily coefficients of variation of $\mathrm{CPP}$ and AIx were $4.3 \pm 3.3 \%$ and $6.1 \pm 4.3 \%$ in our laboratory, respectively [15]. The incident wave height was the value of the difference between the end-diastolic pressure and the first systolic peak pressure (i.e., first systolic peak pressure - DBP).

\subsubsection{Stroke volume and stroke volume index}

Brachial arterial waveforms were recorded using automated oscillometric cuffs (form PWV/ABI, Colin Medical Technology, Komaki, Japan) attached to both arms. Stroke Volume (SV) was calculated from the systolic area (area under the curve of the arterial pressure wave during systole) using BeatScope 1.0 (TNOBiomedical Instrumentation, Amsterdam, The Netherlands), as previously reported [16-18]. The Stroke Volume Index (SVI) was calculated as the SV/body surface area. Body surface area was calculated using the following formula: body surface area = weight $(\mathrm{kg})^{0.444} \times$ height $(\mathrm{m})^{0.663} \times 0.008883$ [19].

\subsubsection{Carotid-femoral pulse wave velocity}

Using two applanation tonometry sensors incorporating an array of 15 transducers (form PWV/ABI, Colin Medical Technology, Komaki, Japan), carotid and femoral artery pulse waves were obtained simultaneously [20]. The pulse transition times between carotid and femoral pulse waveforms were obtained by the footto-foot method (the carotid "foot" to the femoral "foot"). The 
Carotid-femoral Pulse Wave Velocity (cfPWV) was calculated by dividing the distance by transit time. The daily coefficient of variation of cfPWV was $4.5 \pm 3.2 \%$ in our laboratory [15].

\subsubsection{Brachial blood pressure and heart rate}

Using a previously described noninvasive vascular profiling system (form PWV/ABI), brachial SBP (bSBP), bDBP, Brachial Pulse Pressure (bPP), and Heart Rate (HR) were measured [20]. MBP was calculated as bDBP $+(\mathrm{bSBP}-\mathrm{bDBP}) / 3$.

\subsection{Statistical Analysis}

All data are reported as the mean $\pm \mathrm{SD}$. Normality was verified using the Shapiro-Wilk test for each variable. The unpaired $t$-test and chi-square test were used to examine significant differences in characteristics and hemodynamics (central and peripheral) between the two groups (the kendo athlete group and the control group). In addition, analysis of covariance was used to adjust statistically for the influence of potential group differences in body composition and hemodynamics. Correlations of cPP with SVI and $\mathrm{d} P / \mathrm{d} T_{\max }$ were assessed using Pearson's correlation coefficient $(r)$ across all participants. Partial correlation analysis was used to test these correlations adjusting for covariance. Statistical significance was set at $p<0.05$. Analyses were conducted using IBM SPSS Statistics version 26 (IBM Japan Corp., Tokyo, Japan).

\section{RESULTS}

The characteristics and hemodynamics of the participants are shown in Table 1. Body mass and Body Mass Index (BMI) were significantly higher in the kendo athlete group than in the control

Table 1 Characteristics and hemodynamics of the participants

\begin{tabular}{|c|c|c|c|}
\hline & Control & Kendo & \multirow{2}{*}{$p$-value } \\
\hline & $(n=29)$ & $(n=36)$ & \\
\hline Age, years & $21 \pm 2$ & $20 \pm 1$ & 0.02 \\
\hline Women, $n(\%)$ & $12(41)$ & $13(36)$ & 0.66 \\
\hline Height, $\mathrm{cm}$ & $166 \pm 9$ & $169 \pm 9$ & 0.13 \\
\hline Body mass, kg & $59 \pm 13$ & $69 \pm 9$ & $<0.01$ \\
\hline Body mass index, $\mathrm{kg} / \mathrm{m}^{2}$ & $21 \pm 3$ & $24 \pm 2$ & $<0.01$ \\
\hline Brachial systolic $\mathrm{BP}, \mathrm{mmHg}$ & $112 \pm 9$ & $123 \pm 10$ & $<0.01$ \\
\hline Brachial mean BP, mmHg & $81 \pm 7$ & $86 \pm 8$ & 0.01 \\
\hline Brachial diastolic BP, mmHg & $65 \pm 7$ & $67 \pm 8$ & 0.24 \\
\hline Brachial pulse pressure, $\mathrm{mmHg}$ & $47 \pm 6$ & $56 \pm 5$ & $<0.01$ \\
\hline Heart rate, beats/min & $58 \pm 9$ & $53 \pm 6$ & $<0.01$ \\
\hline $\mathrm{AP}, \mathrm{mmHg}$ & $3 \pm 5$ & $5 \pm 8$ & 0.38 \\
\hline $\mathrm{AP}$ adjusted for heart rate, $\mathrm{mmHg}$ & $0.1 \pm 3.7$ & $-0.7 \pm 7.4$ & 0.55 \\
\hline AIx, $\%$ & $9 \pm 11$ & $11 \pm 16$ & 0.60 \\
\hline AIx adjusted for heart rate, $\%$ & $1.1 \pm 10.3$ & $0.4 \pm 14.7$ & 0.82 \\
\hline Central systolic BP, mmHg & $102 \pm 11$ & $115 \pm 11$ & $<0.01$ \\
\hline Incident wave height, $\mathrm{mmHg}$ & $33 \pm 9$ & $42 \pm 11$ & $<0.01$ \\
\hline Stroke volume, mL & $56 \pm 12$ & $73 \pm 14$ & $<0.01$ \\
\hline $\mathrm{cfPWV}, \mathrm{cm} / \mathrm{s}$ & $688 \pm 66$ & $726 \pm 95$ & 0.07 \\
\hline
\end{tabular}

Values are mean \pm SD or frequency counts (\%). Control, control group; Kendo, kendo athlete group; BP, blood pressure; AIx, augmentation index; AP, augmented pressure; cfPWV, carotid-femoral pulse wave velocity. group. Body mass and BMI remained significant after adjusting for age and sex (body mass: $F=17.56, p<0.01$; BMI: $F=15.32$, $p<0.01)$. Height did not differ between the two groups. bSBP, MBP, and bPP were significantly higher in the kendo athlete group than in the control group, whereas bDBP was not significantly different between the two groups. HR, however, was significantly lower in the kendo athlete group than in the control group. cSBP (Cohen's $d=1.22$ ), incident wave height (Cohen's $d=0.91$ ) and SV (Cohen's $d=1.25$ ) in the kendo athlete group were significantly higher than those in the control group. These results remained significant after adjusting for age, sex, BMI, HR, and AP adjusted for HR (cSBP: $F=21.54, p<0.01$, partial $\eta^{2}=0.27$; incident wave height: $F=13.22$, $p<0.01$, partial $\eta^{2}=0.19$; SV: $F=14.93, p<0.01$, partial $\eta^{2}=0.21$ ). cfPWV in the kendo athlete group trended to be higher than those in the control group. On the other hand, AIx and AP did not differ between the groups. AIx adjusted for HR and AP adjusted for HR did not differ when adjusted for age, sex, and height (AIx adjusted for HR: $F=2.07, p=0.16$; AP adjusted for HR: $F=1.23, p=0.27$ ).

Figure 1 shows $c P P, S V I$ and $\mathrm{d} P / \mathrm{d} T_{\max }$ of the two groups. $\mathrm{cPP}$ (Cohen's $d=1.24$ ), SVI (Cohen's $d=0.86$ ) and $\mathrm{d} P / \mathrm{d} T_{\max }$ (Cohen's $d=0.88$ ) were significantly higher in the kendo athlete group than in the control group. These results remained significant after adjusting for age, sex, BMI, HR, and AP adjusted for HR (cPP: $F=14.77, p<0.01$, partial $\eta^{2}=0.20$; SVI: $F=11.46, p<0.01$, partial $\eta^{2}=0.17 ; \mathrm{d} P / \mathrm{d} T_{\max }: F=12.56, p<0.01$, partial $\left.\eta^{2}=0.18\right)$.

The correlations of $\mathrm{CPP}$ with SVI and $\mathrm{d} P / \mathrm{d} T_{\max }$ across all participants are shown in Figure 2. SVI and $\mathrm{d} P / \mathrm{d} T_{\max }$ showed a significant positive correlation with $\mathrm{CPP}$. These relationships remained significant after adjusting for age, sex, BMI, HR, AP adjusted for $\mathrm{HR}$, and cfPWV (SVI: partial $r=0.30, p<0.05 ; \mathrm{d} P / \mathrm{d} T_{\max }$ : partial $r=0.76, p<0.01)$.

\section{DISCUSSION}

The present cross-sectional study compared the central BP of highlevel university kendo athletes and age-matched controls without exercise habits. cSBP and cPP were significantly higher in the kendo athlete group than in the control group. SVI and $\mathrm{d} P / \mathrm{d} T_{\max }$ in the kendo athlete group were also significantly higher than those in the control group, and these showed a significant positive correlation with $\mathrm{CPP}$. These results suggest that habitual kendo training may increase central pulse pressure which may be associated with kendo training-induced adaptation of the left ventricle.

Previous studies have suggested that the adaptation of central BP depends on the types of exercise training [9,21]. However, the effects of habitual kendo training on central BP have not been established. In this cross-sectional study, cSBP and cPP in the kendo athlete group were significantly higher than those in the control group. These results suggest that habitual kendo training increases central BP and pulsatile pressure.

Central BP is strongly modulated by reflected waves [1]. We have reported that the average AP, a wave reflection index, was in the range -2 to $0 \mathrm{mmHg}$ in young adults $[15,22]$. It has been considered that this phenomenon in young adults was caused by geometry and stiffness of peripheral muscular arteries and/or arterioles at the major reflecting sites [23]. Therefore, central BP in young adults is thought to be more strongly affected by forward pressure waves than reflected waves. Consistent with our previous 

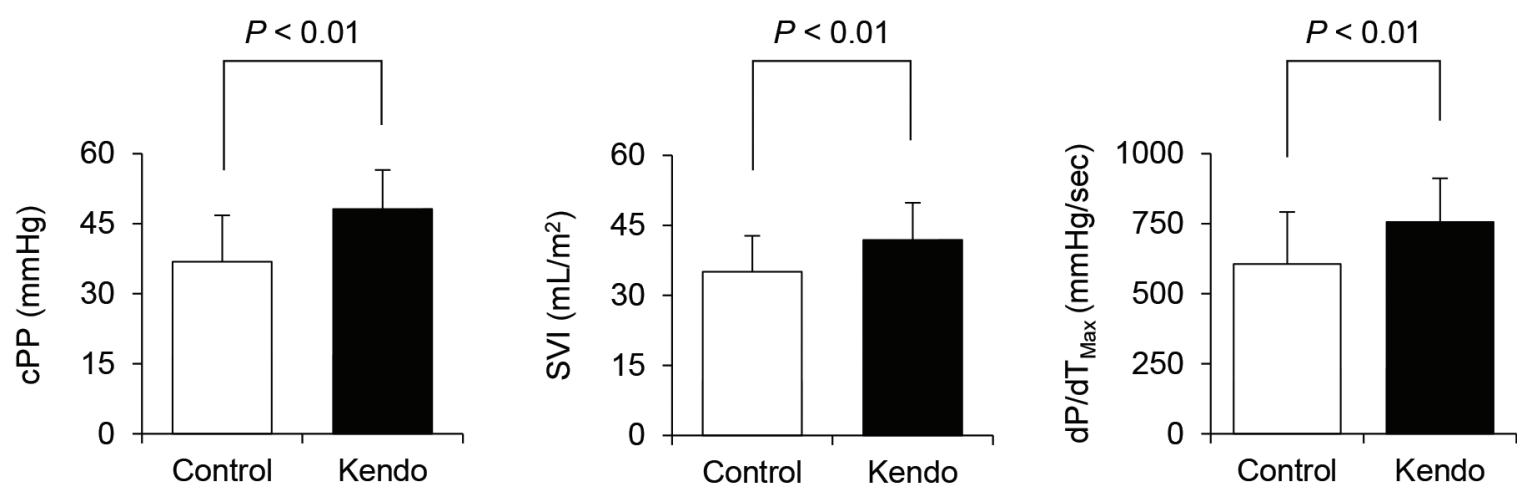

Figure 1 Central pulse pressure (cPP) (a), stroke volume index (SVI) (b), and $\mathrm{d} P / \mathrm{d} T_{\max }$ (c) in the kendo athlete group and the control group. Data are presented as the mean $\pm \mathrm{SD}$.
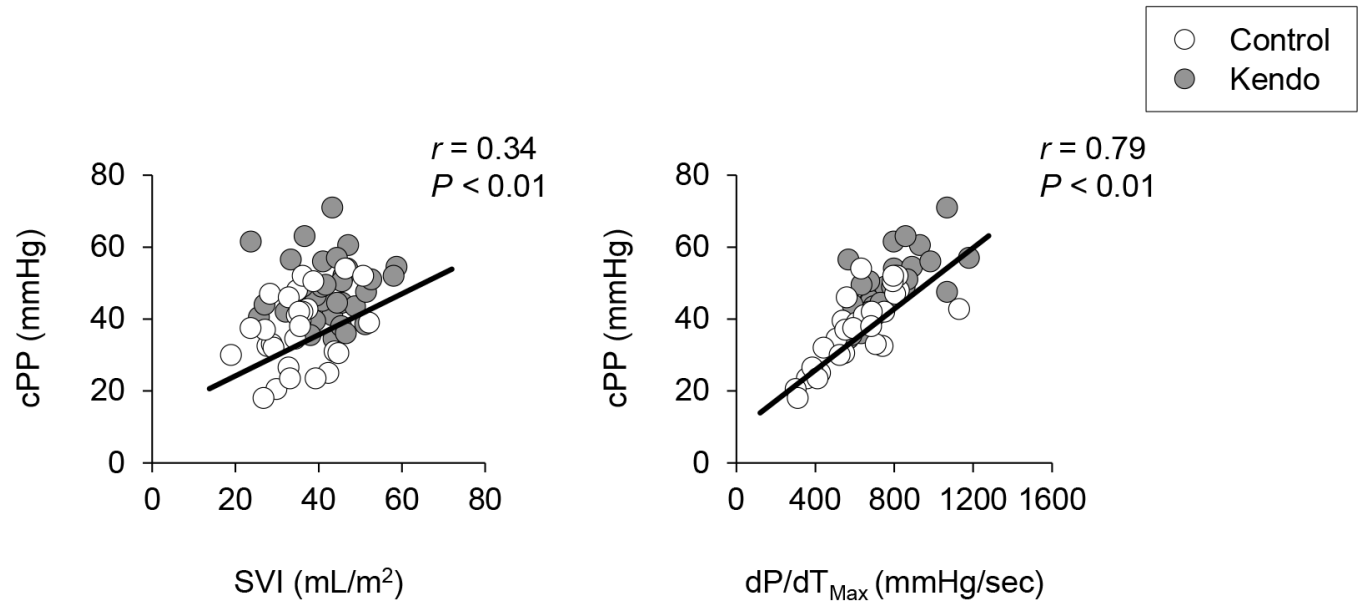

Figure 2 The relationships between central pulse pressure (cPP) with stroke volume index (SVI) (a) and $\mathrm{d} P / \mathrm{d} T_{\max }$ (b) in all participants (the kendo athlete group and the control group).

study, APs in the kendo athlete group and the control group were $0.1 \pm 3.7$ and $-0.7 \pm 7.4 \mathrm{mmHg}$, respectively, in the current study. In contrast, central BP, SVI and $\mathrm{d} P / \mathrm{d} T_{\max }$ were all significantly higher in the kendo athlete group than in the control group. Furthermore, SVI and $\mathrm{d} P / \mathrm{d} T_{\max }$ showed a significant positive correlation with cPP. Our results suggest that augmentation in central BP induced by habitual kendo training results from forward pressure waves, but not reflected waves.

Because of the different effects of anaerobic exercise training and resistance training on central $\mathrm{BP}$, it is of particular interest to determine how BPs adapt to the combination of these training types. Kendo is ideal for investigating of training adaptation since it induces the characteristics of anaerobic exercise training and resistance training; that is, kendo athletes had both high anaerobic capacity and high muscle strength compared with sedentary controls [14]. Interestingly, the present study suggests that left ventricular systolic function in the kendo athletes was higher than that in the sedentary controls (as seen in anaerobic exercise training) [24]. As a result, the anaerobic exercise training component appears to outweigh the resistance training component, producing higher SVI and $\mathrm{d} P / \mathrm{d} T_{\max }$ in kendo. However, central $\mathrm{BP}$ was higher in the kendo athlete group than in the control group (as not seen in anaerobic exercise training), and the increased central $\mathrm{BP}$ with habitual kendo training was associated with SVI and $\mathrm{d} P / \mathrm{d} T_{\max }$. These results suggest that habitual kendo training may increase central BP through kendo training-induced adaptation in the left ventricle, in contrast to anaerobic exercise training alone and resistance training alone.

Normally, central elastic arteries (e.g., aorta and carotid artery) effectively buffer cardiac pulsatile fluctuation generated from the left ventricle [1]. Therefore, aortic stiffness may modulate the relationship between changed cardiac ejection and central BP with habitual kendo training. In the present study, cfPWV, which was measured as aortic stiffness, trended to be higher in the kendo athlete group than in the control group. However, the correlations of cPP with SVI and $\mathrm{d} P / \mathrm{d} T_{\max }$ remained significant after adjusting for cfPWV. These results suggest that increased central BP with habitual kendo training was caused by adaptation in the left ventricle independent of aortic stiffness.

As well as central BP, brachial BP (bSBP and bPP) was significantly higher in the kendo group than in the control group. The arterial tree has mainly two functions; first, to deliver blood from the left ventricle to bodily organs and tissues, and second, to cushion the pulsations generated by the heart $[1,2]$. In the present study, incident wave height and $\mathrm{d} P / \mathrm{d} T_{\max }$, which was measured as left ventricle systolic function, were significantly higher in the kendo athlete group than in the control group. On the other hand, AIx, which 
was measured as left ventricular afterload, did not differ between the two groups. These results suggest that larger pulsations are not absorbed in the aorta and so extend down into the peripheral circulation (i.e., brachial arteries) in the kendo athletes [4].

There are several important limitations to the present study. First, we cannot detect cause and effect relations because the baseline for the kendo athletes and the controls is not known. Second, we studied an only apparent small number of participants. Longitudinal studies or interventional studies with a larger sample size are necessary to determine the adaptations of central BP in kendo athletes. Third, we were unable to assess the anaerobic capacity and muscular strength of the participants. Further studies are still needed how combined anaerobic exercise and muscular strength training changes central BP. Forth, brachial arterial waveforms may have measurement errors because different muscular physique between the kendo athlete group and the control group affects blood pressure determination using an oscillometric device. Fifth, we could not perfectly match the sex of the two groups. However, sex would not affect because the present results did not any change after the consideration of various confounders including sex.

\section{CONCLUSION}

In summary, central BP (cSBP and cPP) was significantly higher in the kendo athlete group than in the control group. SVI and $\mathrm{d} P / \mathrm{d} T_{\max }$ but not AP in the kendo athlete group were also significantly higher than those in the control group. In addition, SVI and $\mathrm{d} P / \mathrm{d} T_{\max }$ showed a significant positive correlation with central BP. These results suggest that habitual kendo training may increase central BP and that this is related to regular kendo traininginduced adaptation of the left ventricle and forward pressure wave, but not reflected waves.

\section{CONFLICTS OF INTEREST}

The authors have no financial, consultant, institutional, or other relationships that might lead to bias or a conflict of interest.

\section{AUTHORS' CONTRIBUTION}

YT, TS, CY and SM conceived and designed research. MY, YT, TS, JP and RM conducted experiments. JS contributed new analytical tools. MY and KT analyzed data. MY wrote the manuscript. KT, JS and SM revised the manuscript. All authors read and approved the manuscript.

\section{FUNDING}

No financial support was provided.

\section{ACKNOWLEDGMENTS}

The authors would like to thank all participants in the study. We also thank our (S.M.s) laboratory members at University of Tsukuba for technical assistance.

\section{ABBREVIATIONS}

AIx, augmentation index; AP, augmented pressure; bDBP, brachial diastolic blood pressure; BMI, body mass index; BP, blood pressure; bPP, brachial pulse pressure; bSBP, brachial systolic blood pressure; cfPWV, carotid-femoral pulse wave velocity; $c \mathrm{PP}$, central pulse pressure; cSBP, central systolic blood pressure; HR, heart rate; MBP, mean blood pressure; SV, stroke volume; SVI, stroke volume index.

\section{REFERENCES}

[1] Nichols WW, O'Rourke MF, Vlachopoulos C. McDonald's Blood Flow in Arteries: Theoretical, Experimental and Clinical Principles. 6th ed., London, UK: Hodder Arnold; 2011.

[2] Safar ME, London GM. Therapeutic studies and arterial stiffness in hypertension: recommendations of the European Society of Hypertension. The Clinical Committee of Arterial Structure and Function. Working Group on Vascular Structure and Function of the European Society of Hypertension. J Hypertens 2000;18:1527-35.

[3] Belz GG. Elastic properties and Windkessel function of the human aorta. Cardiovasc Drugs Ther 1995;9:73-83.

[4] O’Rourke MF, Hashimoto J. Mechanical factors in arterial aging: a clinical perspective. J Am Coll Cardiol 2007;50:1-13.

[5] Williams MA, Haskell WL, Ades PA, Amsterdam EA, Bittner V, Franklin BA, et al. Resistance exercise in individuals with and without cardiovascular disease: 2007 update: a scientific statement from the American Heart Association Council on Clinical Cardiology and Council on Nutrition, Physical Activity, and Metabolism. Circulation 2007;116:572-84.

[6] Kawamoto R, Kohara K, Katoh T, Kusunoki T, Ohtsuka N, Abe M, et al. Effect of weight loss on central systolic blood pressure in elderly community-dwelling persons. Hypertens Res 2014; 37:933-8

[7] Clark T, Morey R, Jones MD, Marcos L, Ristov M, Ram A, et al. High-intensity interval training for reducing blood pressure: a randomized trial vs. moderate-intensity continuous training in males with overweight or obesity. Hypertens Res 2020;43:396-403.

[8] Hanssen H, Minghetti A, Magon S, Rossmeissl A, Papadopoulou A, Klenk C, et al. Superior effects of high-intensity interval training vs. moderate continuous training on arterial stiffness in episodic migraine: a randomized controlled trial. Front Physiol 2017;8:1086

[9] Bertovic DA, Waddell TK, Gatzka CD, Cameron JD, Dart AM, Kingwell BA. Muscular strength training is associated with low arterial compliance and high pulse pressure. Hypertension 1999;33:1385-91.

[10] All Japan Kendo Federation. Available from: https://www.kendo. or.jp/ (accessed January 5, 2021).

[11] International Kendo Federation. Available from: https://www. kendo-fik.org/ (accessed January 5, 2021).

[12] Hayashi K, Horiyama K, Iho K, Washimi K, Nakata M. Longitudinal changes of upper limb, lower limb and trunk muscle strength in kendo players. Res J Budo 1993;25:21-9 (in Japanese).

[13] Kendo Renmei ZN. The official guide for kendo instruction. Tokyo, Shusansha: All Japan Kendo Federation; 2011. 
[14] Katsuki T, Demura S, Tanabe M. Physical characteristics of college male kendo competitors. Res J Budo 1993;26:15-24 (in Japanese).

[15] Tagawa K, Takahashi A, Yokota A, Sato T, Maeda S. Aortic diastolic pressure decay modulates relation between worsened aortic stiffness and myocardial oxygen supply/demand balance after resistance exercise. J Appl Physiol 2019;127:737-44.

[16] Sugawara J, Tanabe T, Miyachi M, Yamamoto K, Takahashi K, Iemitsu $\mathrm{M}$, et al. Non-invasive assessment of cardiac output during exercise in healthy young humans: comparison between Modelflow method and Doppler echocardiography method. Acta Physiol Scand 2003;179:361-6.

[17] Wesseling KH, Jansen JR, Settels JJ, Schreuder JJ. Computation of aortic flow from pressure in humans using a nonlinear, three-element model. J Appl Physiol 1993;74:2566-73.

[18] Wesseling KH, de Wit B, Weber JAP, Smith NT. A simple device for the continuous measurement of cardiac output. Its model basis and experimental verification. Adv Cardiovasc Phys 1983;5:16-52.

[19] Fujimoto S, Watanabe T, Sakamoto A, Yukawa K, Morimoto K. [Studies on the physical surface area of Japanese. 18. Calculation formulas in three stages over all ages]. Jap J Hyg 1968;23:443-50 (in Japanese).

[20] Sugawara J, Hayashi K, Yokoi T, Tanaka H. Age-associated elongation of the ascending aorta in adults. JACC Cardiovasc Imaging 2008;1:739-48.

[21] Nualnim N, Barnes JN, Tarumi T, Renzi CP, Tanaka H Comparison of central artery elasticity in swimmers, runners, and the sedentary. Am J Cardiol 2011;107:783-7.

[22] Tagawa K, Choi Y, Ra SG, Yoshikawa T, Kumagai H, Maeda S. Resistance training-induced decrease in central arterial compliance is associated with decreased subendocardial viability ratio in healthy young men. Appl Physiol Nutr Metab 2018; 43:510-16.

[23] Nichols WW. Clinical measurement of arterial stiffness obtained from noninvasive pressure waveforms. Am J Hypertens 2005; 18:3S-10S

[24] Mahdiabadi J, Gaeini AA, Kazemi T, Mahdiabadi MA. The effect of aerobic continuous and interval training on left ventricular structure and function in male non-athletes. Biol Sport 2013;30:207-11 